\title{
Analysis of Aviation Industry for Aggressive Decision Making
} doi : https://doi.org/10.32628/CSEIT2173134

\section{Mohmmed Ali Asgar Niazi ${ }^{1}$, Dr. Sheikh Fahad Ahmad ${ }^{2}$}

1M.Tech Scholar, Department of Computer Science and Engineering, Integral University Lucknow, India

${ }^{2}$ Assistant Professor, Department of Computer Science and Engineering, Integral University Lucknow, India

\section{Article Info}

Volume 7, Issue 3

Page Number: 625-631

\section{Publication Issue :}

May-June-2021

Article History

Accepted : 10 June 2021

Published : 16 June 2021

\section{ABSTRACT}

Big Data Analytics is very useful for the business users and data scientists. It is very useful to take better, faster and right decision for the organization. Organizations and individuals should exhibit the circumspection while utilizing Big Data. In this work we intend to develop a methodology for getting ethical access of big data and ethically scrutinize it to attain the business objectives. We consider the case study of aviation sector, formulate some questions to upraise the system. We attain the ethical permission from twitter for this purpose. We consider the tweets of general public as they were posted in public areas and falls under informed consent category."

Keywords: Big Data, Opinion mining, Business Analysis

\section{INTRODUCTION}

Big Data analytics is an area of computer science which defines an ability to analyze varieties of data such as structured, semi-structured and unstructured. Every day we generate and consume data. We all know that data is growing very speedy with $40 \%$ annual rate, and we will reach to about 45 Zetta bytes of data by 2020. If you talk about 2010 then at that time it was 1.2 trillion GB of data was generated. This amount of data has doubled to 2.4 trillion gigabytes in year 2012 and to about 5 trillion gigabytes in year 2014[4].

With fast innovations, frequent advancement of technology and an unexpectedly growing internet populace, structures and corporations are producing huge quantities of statistics to the melody of terabytes and even petabytes of facts. It is fact that facts being generated in very huge volumes with tremendous velocity in all multi-dependent formats like images, motion pictures, weblogs, sensor information, and so on. From all diverse sources, there may be a big call for to correctly keep and examine this giant quantity of information to make it utilizable.

\section{$\begin{array}{llllll}0 & 10 & 15 & 20 & 2530354045\end{array}$ GB of Data (In Trillions)}

Figure 1: Growth of Data 
Huge amounts of data we are creating every day. We are playing role of information consumer as well as producer nowadays. Analysis of this huge data within minimum time is challenging nowadays, not only faster output but we also need accurate information of different varieties of data. Whenever we talked about big data we must cover 3Vs of Big Data that is Variety, Velocity and Volume of data[5].

\section{LITERATURE REVIEW}

Big data analytics is an emerging trend for the research scholar, academicians, organizations and it has created an impact that it will be able to resolve the data related issues of any organization.

Big data analytics[1] is a procedure of gathering, forming and investigating huge amount of data ("big data") which is used to determine designs and further valuable info. Big data is not only used to extract the meaningful information from the data, but it also helps to extract the data that is essential for the any organization conclusions. Big data analysts mostly extract the information that originates from investigating the facts[6].

Many researchers have analyzed the diversities of data. Some of the researchers has analyzed GPS data, some of the researches working on satellite data, few working on longitude data. Some of the researchers has studied the social media data. Before few months back one survey came that facebook become the users with the 2 Billion. Many people are doing marketing on social media. It plays a key role for the decision perception. Because, after analysis of social media data we can identified the perception of human behaviours and that can be used by the organization for effective decision. Social media data is nowadays analyzed by research scholars, investigators, academicians, medias and organizations[2][7].
Many organization and researchers emphasized o $\mathrm{n}$ "Big Data" because it is a comprehensive and $\mathrm{i}$ ntellectual perception and it is highly recommen ded by the scholars and investigators. It is widel $y$ used technique that can be used to take right decision in favour of organizations strategy, whic $h$ quickly returns the perfect result from the ma ssive quantities of the data, which can be in any formats like CCTV footage, weblogs, social medi a posts etc, and stored on any geography locatio $\mathrm{ns}[3]$.

\section{PROPOSED METHODOLOGY}

Our proposed approach is divided in two phases.

\section{Phase 1: Planning}

Step 1: Discuss with experts and decision makes to def ine the business objectives and make the discus sion with experts unless the objectives are clear ly defined.

Step 2: When the objectives have been determined cl early, identify the stakeholders that would be i nvolved in attaining the objectives.

Step 3: Frame ethical questions that must be answere $\mathrm{d}$ to attain the objectives

\section{Phase 2: Evolution}

Step 1 : Acquire the ethical approval from social medi a from where data is to be collectd Generally approval not given in cases of high ri sk area of research, threats to security, disturba nce of hormonal integrity of society, un aware of negative connotations of \# they are then labelled as 'racist', nudity etc.

Step 2: If approval is not found, reframe the ethical ev aluation questions. If found review and revise $\mathrm{d}$ 
ifferent data collection tools and also determine the correct hashtags.

Step 3: Obtain the consent from concerned stakehold ers

- Find out what the user consented to at the tim e of data capture? Terms and conditions.

- Is the data 'open access'

- why is it being passed on? Need to be clear abo ut this

- Context of posts/tweets is important or may be deleted. Need to address this.

- Is the data from a 'real' identity?

-Too many "participants" (Informed consent)

Step 4: Collect social media data

\section{Phase 3: Decision Making}

Step 1: Perform Ethical Analysis of data gathered fro m social media

\section{$>$ Pre-processing of Data(Posts/tweets)}

○ Removing Multiple tweets from same person

○ Removing weblinks

- Removing special symbols

○ Removing English stopwords

$>$ Calculating the score of each Posts/ tweets

The approach here is to estimate the affirmative and depressing words in each tweet /post and set the confidence level. In this way, we can confirm the affirmative or depressing extent of the twee. However, there are manifold ways to compute these points; here is one formula for performing such calculations.

- $\quad$ result $=$ Number of affirmative words -

Number of depressing words

$\circ$ If result $>0$, then the tweet/post has encouraging reaction

○ If result $<0$, then that the tweet/post has unenth usiastic reaction $\circ$ If result $=0$, then that the tweet/post has disinter ested reaction"

$>$ Representing analysis using graphs

Step 2: Answer the questions framed in step 3 of phas

e 1 on the basis of positive, negative and neutra 1 reactions and plotted graphs.

Step 3: Utilize the knowledge in attaining business ob jectives

\section{EXPERIMENTAL EVALUATION}

We considered the case study of aviation sector, formulate some questions to upraise the system. We attain the ethical permission from twitter for this purpose. We consider the tweets of general public as they were posted in public areas and falls under informed consent category. We implemented our proposed methodology in R Language.

As we considered the case study of aviation system, we derived some questions as follows:

$\circ$ Which airline is being rated highly by customers?

- What is the rating pattern of customers?

$\circ$ What are the problems being faced by customers?

- What are the facilities being expected by customers?

With the proper consent of twitter we performed ethical analysis to attain business objectives. Generated results are as follows: 


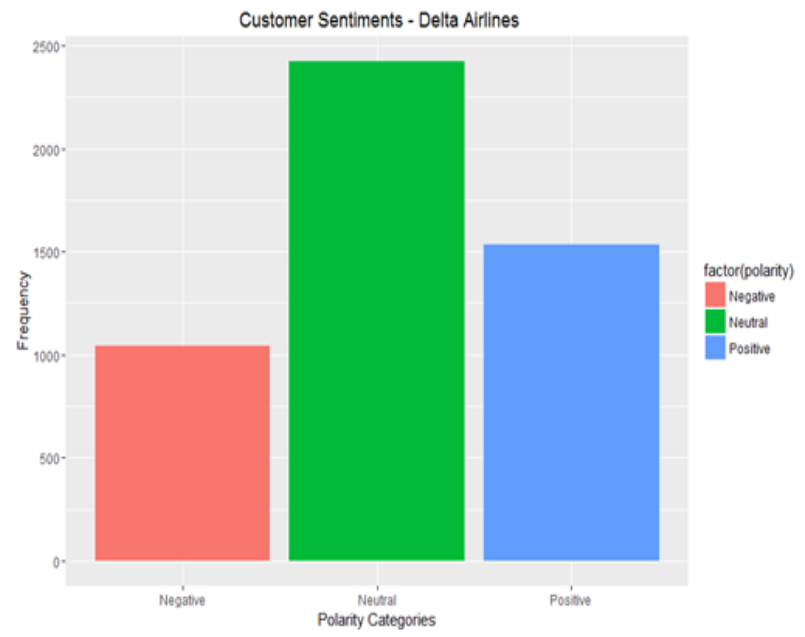

Figure 2: Customer Sentiments (Delta Airlines)

The bar graph shown in figure 2 depicts divergence, if we intimately analyze the graph; it divulge that among 5,000 twitter users, 1,100 twitter users have commented in a pessim istic way, 2,380 users are on middle-of-theroad. Nevertheless, 1,520 users are pretty affirmat ive about the airline.

\section{Customer reaction Scores (Delta Airlines)}

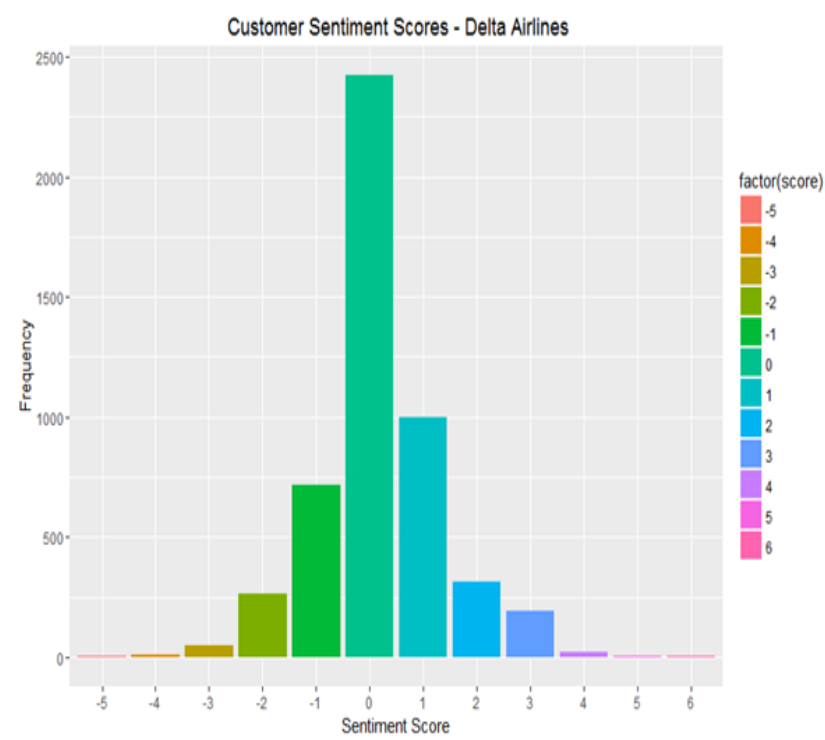

Figure 3: Customer Sentiments Scores (Delta Airlines) The bar graph shown in figure 3 portrays twitter user's sentiment score, pessimis tic score symbolized by the () symbol, which designate despondency of users with the airline, while the affirmative score indic ate that users are blissful with the airline. Zero c haracterizes that twitter users are neutral.

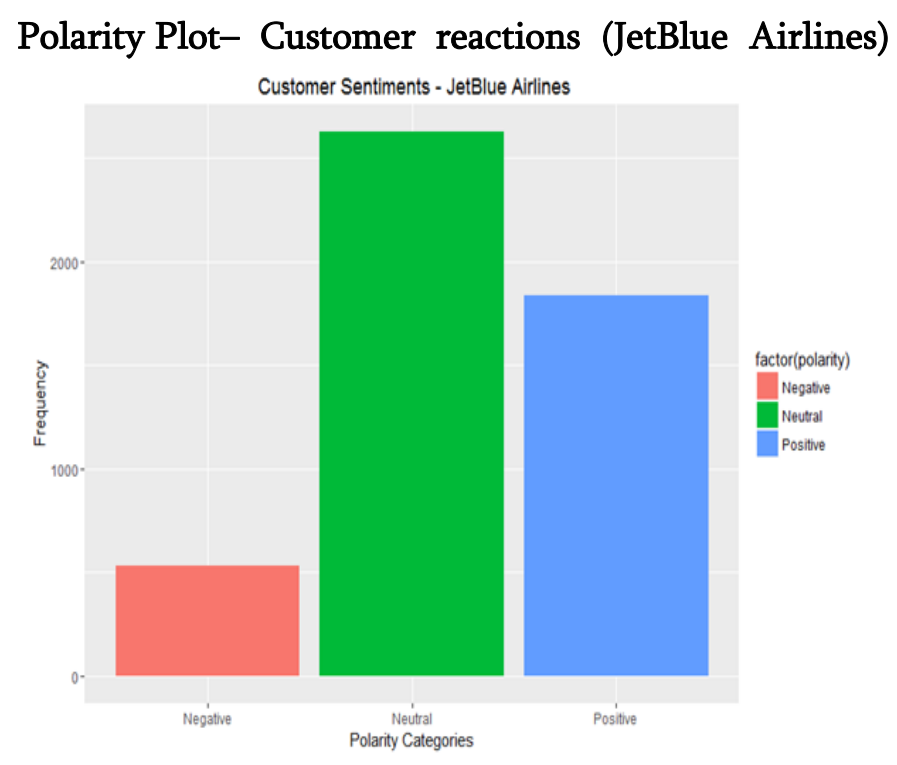

Figure 4: Customer reactions (JetBlue Airlines)

The bar graph shown in figure 4 represents polarity. In this case, among 5,000 t witter users, 550 users have commented pessimisti cally, 2,700 users stay neutral, whereas 1,750 user $\mathrm{s}$ are optimistic about the airline.

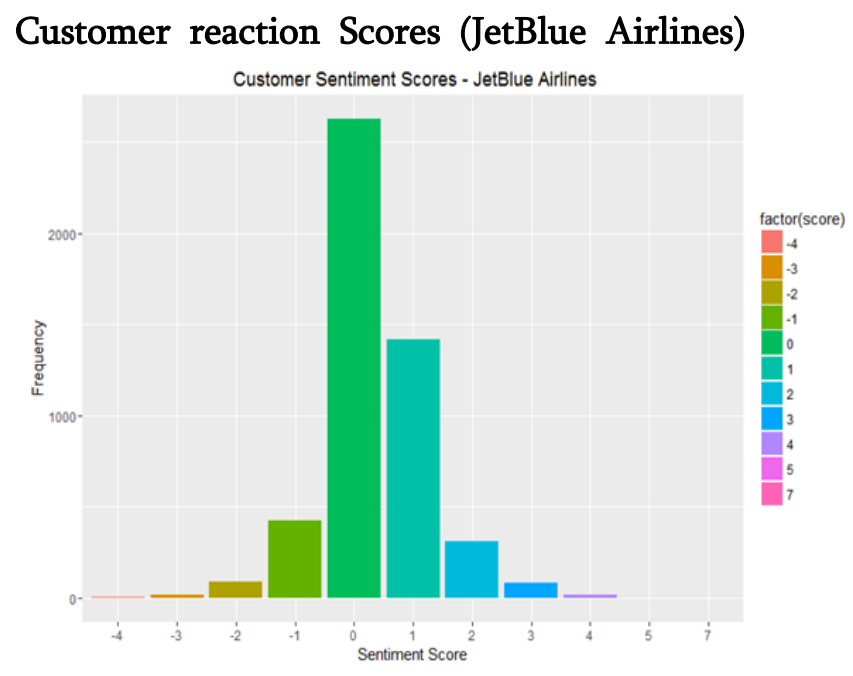

Figure 5: Customer sentiments Scores (JetBlue Airlines)

The bar graph of figure 5 depicts twitter user's sentiment score, negative score denoted by the (-) symbol, which indicates unhappiness with the airline, 
whereas the positive score denotes that users are quite happy. Whereas, zero here represents that users are neutral.

\section{Polarity Plot}

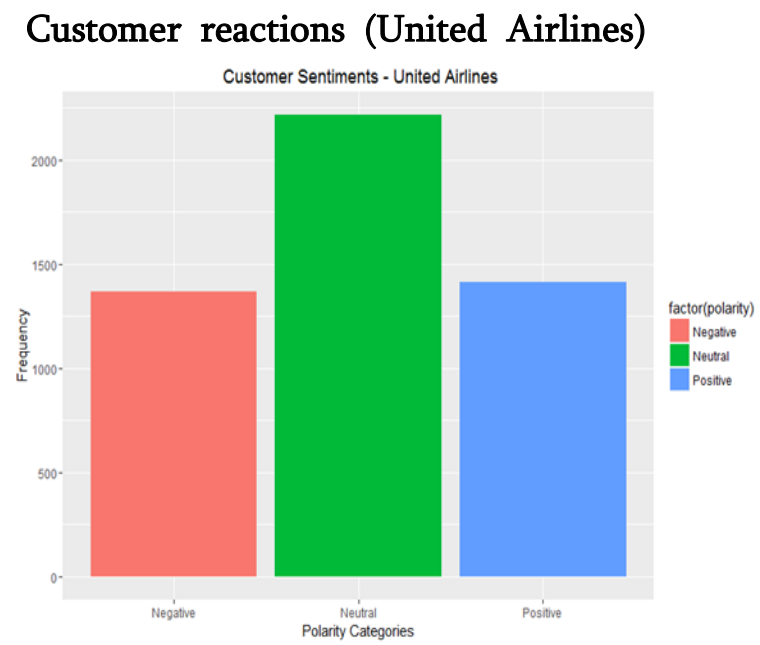

Figure 6: Customer sentiments (United Airlines)

The bar graph of figure 6 represents polarity. In this case, out of the 5,000 twitter users, 1,350 users have commented negatively, whereas 2,200 users are neutral and remaining 1,450 users remain positive about the airline.

\section{Customer Sentiment Scores (United Airlines)}

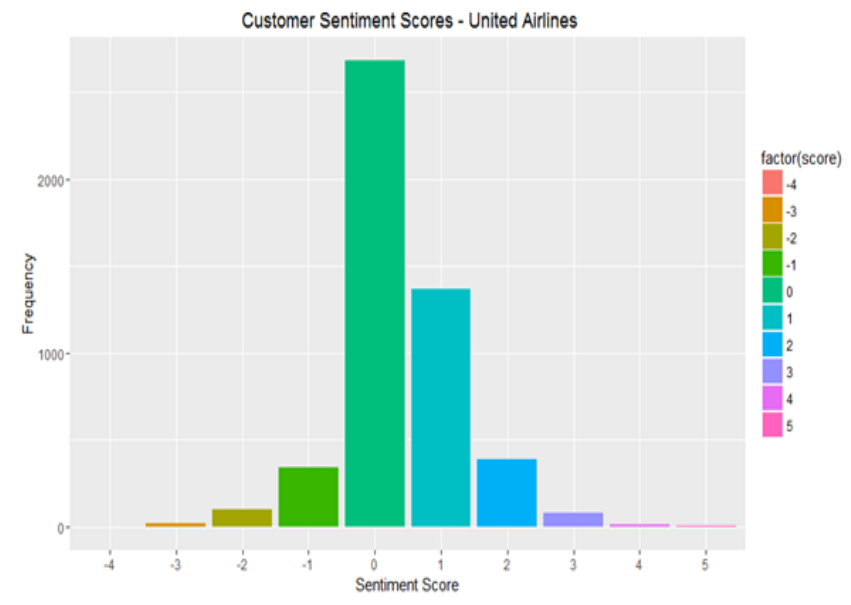

Figure 7: Customer sentiments scores (United Airlines)

The bar graph of figure 7 depicts twitter user's sentiment score, negative score denoted by the (-) symbol indicates unhappiness of users with the airline, whereas the positive score denotes that users are quite happy. While, zero represents that users are neutral about their opinion.

\section{Comparison Charts}

\section{Positive Comparative Analysis}

The pie chart below represents positive percentag e score of these airlines.

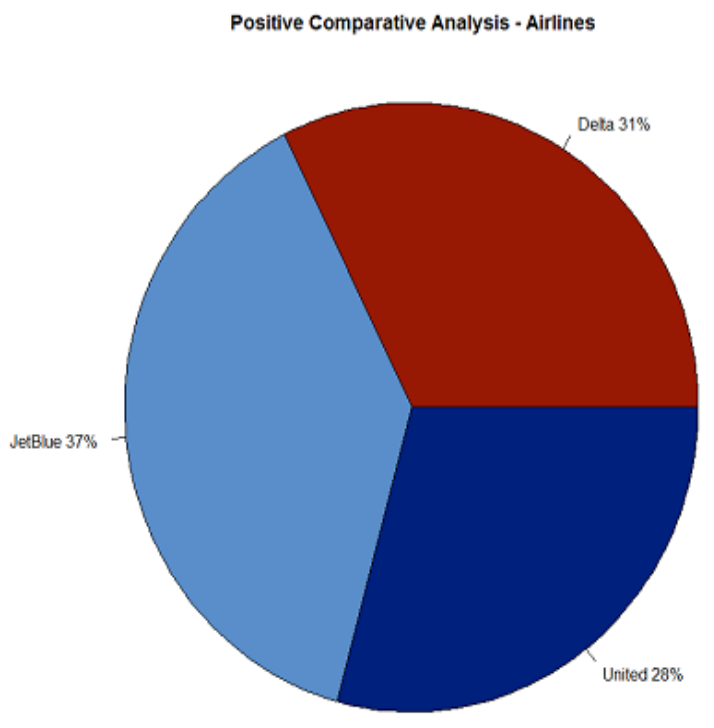

Figure 8: Positive Comparative analysis

\section{Negative Comparative Analysis}

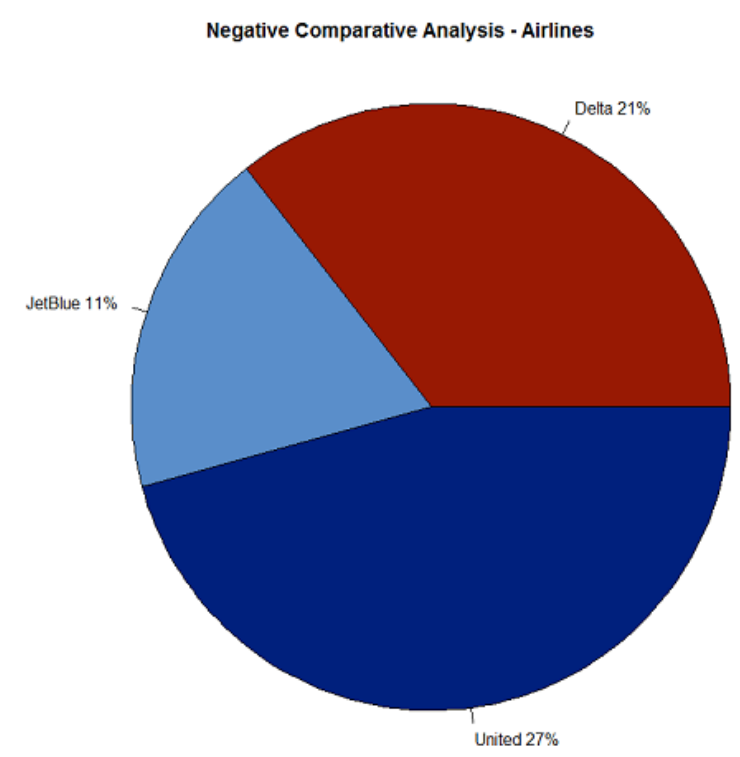

Figure 9 : Negative Comparative analysis 


\section{Neutral Comparative Analysis}

The pie chart below represents neutral percentage score of these three airlines.

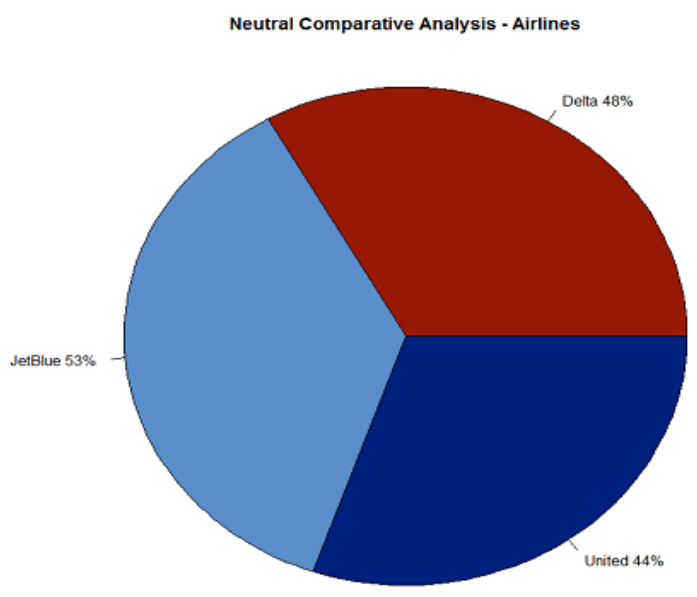

Figure 10 : Neutral Comparative analysis

Thus by using above analysis any business objecti ves van be attained.

\section{Aspect Extraction}

Aspects such as Employee Courtesy, Reliability of Customer Service, Attention to Passengers, Airline Staff Image, Flight Attendant promptness and service, Interest in solving customers problems, are considered in the study as shown in fig 11 .

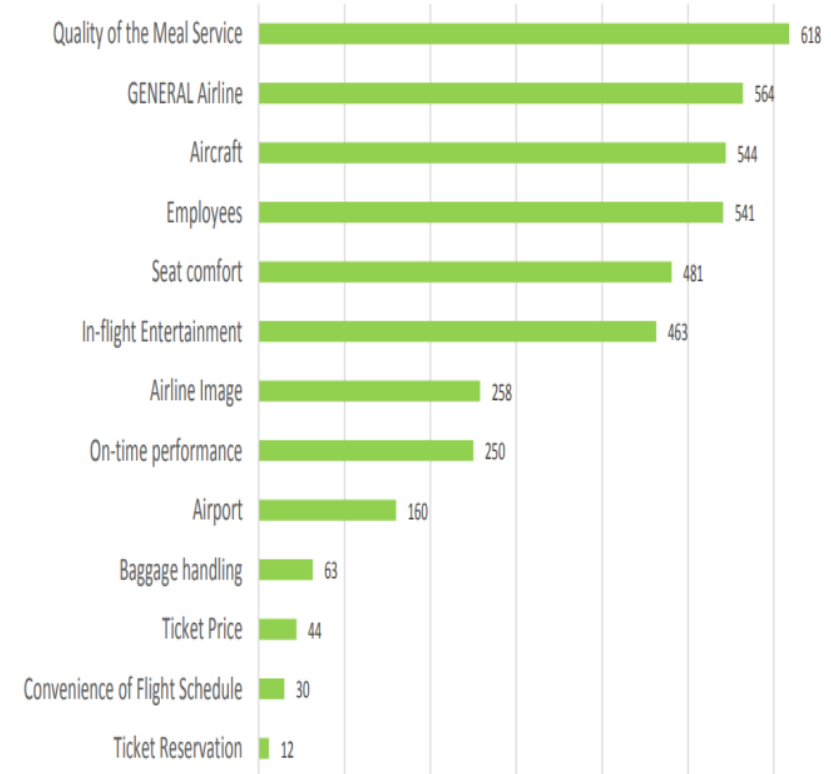

Figure 11: Aspect Analysis

\section{Comparison and evaluation}

The performance of various sentiment analysis techniques was measured on the basis of accuracy. That is, what percentage of text was accurately classified by the sentiment analysis technique? The performance of different studies discussed earlier are represented in fig 12 .

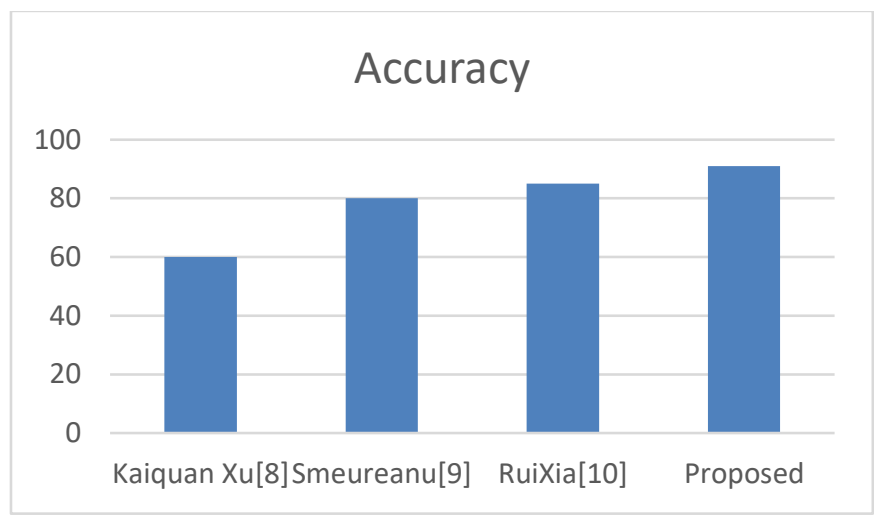

Figure 12: Comparative Chart

\section{Conclusion}

In this work, we aimed to devise a methodology for obtaining moral access to large data and morally analyzing it to achieve business objectives. We took the case study for the aviation sector and formulated some questions to upgrade the system. We have obtained a moral permission from Twitter for this purpose.

We looked at public tweets where they were posted i $\mathrm{n}$ public areas and fall under the category of prior app roval. We consider the use of leading US aircraft (Del ta, JetBlue and United Airlines) to demonstrate the fu ndamentals of ethical interaction analysis. By using $t$ he various steps in the proposed curriculum, we cond ucted a moral analysis and generated answers to preformulated questions. 


\section{REFERENCES}

[1]. Zeynep Tufekci, Big Questions for Social Media Big Data: Representativeness, Validity and Other Methodological Pitfalls, Proceedings of the Eighth International AAAI Conference on Weblogs and Social Media.

[2]. Berman, J.J Principles of Big Dat Information. Waltham, Elsevier.

[3]. Walunj Swapnil K, Big Data: Characteristics, Challenges and Data Mining, International Conference on Advances in Information Technology and Management ICAIM 2016

[4]. Dr. Birendra Goswami, Pradip Kumar Chandra "The Evolution Of Big Data As A Research And Development" International Journal of Scientific Research and Engineering Studies (IJSRES) Volume 2 Issue 3, March 2015 ISSN : 2349-8862

[5]. Thiago Poleto, Victor Diogh o Heuer de Carvalho, and Ana Paula Cabral Seixas Costa, The Roles of Big Data in the Decision Support Process: An Empirical Investigation, Springer International Publishing Switzerland 2015.

[6]. Lukoianova, T \& Rubin, V 2014, Veracity Roadmap Is Big Data Objective, Truthful and Credible? Advances in Classification Research Online, Vol. 24, no. 1, doi:10.7152/acro.v24i1.1467.

[7]. Sanger, Johannes, Christian Richthammer, Shoaib Hassan \& Gunther Pernul, „Trust and big data: A roadmap for research ${ }^{\text {ee }}$, In Database and Expert Systems Applications (DEXA), 2014 25th International Workshop on IEEE, pp. 278-282.

[8]. KaiquanXu , Stephen Shaoyi Liao , Jiexun Li, Yuxia Song, "Mining comparative opinions from customer reviews for Competitive Intelligence", Decision Support Systems 50 (2011) 743-754.
[9]. Ion SMEUREANU, Cristian BUCUR, “Applying Supervised Opinion Mining Techniques on Online User Reviews", InformaticaEconomică vol. 16 , no. $2 / 2012$.

[10]. Rui Xia, ChengqingZong, Shoushan Li, "Ensemble of feature sets and classification algorithms for sentiment classification", Information Sciences 181 (2011) 1138-1152.

\section{Cite this article as :}

Mohmmed Ali Asgar Niazi, Dr. Sheikh Fahad Ahmad, "Analysis of Aviation Industry for Aggressive Decision Making", International Journal of Scientific Research in Computer Science, Engineering and Information Technology (IJSRCSEIT), ISSN : 24563307, Volume 7, Issue 3, pp.625-631, May-June-2021. Available at

doi : https://doi.org/10.32628/CSEIT2173134 Journal URL : https://ijsrcseit.com/CSEIT2173134 